\title{
Correction: ESHG warns against misuses of genetic tests and biobanks for discrimination purposes
}

Francesca Forzano - Maurizio Genuardi $($ Yves Moreau $\cdot$ On behalf of the European Society of Human Genetics

Published online: 19 February 2021

(C) The Author(s), under exclusive licence to European Society of Human Genetics 2021

Correction to: European Journal of Human Genetics https://doi.org/10.1038/s41431-020-00786-6

The original version of this paper was inadvertently published with an error in the affiliation of the author Maurizio Genuardi.
The correct affiliations are: Fondazione Policlinico Universitario IRCCS, Rome, Italy and

Dipartimento di Scienze della Vita e Sanità Pubblica, Università Cattolica del Sacro Cuore, Rome, Italy

The original article has been corrected. We apologise for any inconvenience caused to our readers. 